\title{
Laughter's Influence on the Intimacy of Self-Disclosure
}

\section{A.W.Gray, B. Parkinson \& R.I.M. Dunbar}

\section{Experimental Psychology}

\section{University of Oxford}

\begin{abstract}
If laughter functions to build relationships between individuals, as current theory suggests, laughter should be linked to interpersonal behaviours that have been shown to be critical to relationship development. Given the importance of disclosing behaviours in facilitating the development of intense social bonds, it is possible that the act of laughing may temporarily influence the laugher's willingness to disclose personal information. We tested this hypothesis experimentally by comparing the characteristics of self-disclosing statements produced by those who had previously watched one of three video clips that differed in the extent to which they elicited laughter and positive affect. The results show that disclosure intimacy is significantly higher after laughter than in the control condition, and suggest that this effect may be due, at least in part, to laughter itself and not simply to a change in positive affect. However, the disclosure intimacy effect was only found for observer-ratings of participants' disclosures and was absent in the participants' own ratings. We suggest that laughter increases people's willingness to disclose, but that they may not necessarily be aware that it is doing so.
\end{abstract}

Keywords: Laughter; Self-Disclosure; Social relationships; Endorphins; Social bonding 


\section{Introduction}

If laughter functions to build relationships between individuals (Bachorowski \& Owren, 2001; Dunbar, 2012; Li et al, 2009; Owren \& Bachorowski, 2003), it should also be linked to interpersonal behaviours otherwise involved in relationship development (Mehu, 2011). Given the importance of disclosing behaviours in forging affiliations (Collins \& Miller, 1994), it is possible that the act of laughing may temporarily influence the laugher's willingness to disclose personal information. This would certainly be beneficial to the formation of intense social bonds, and hence in line with laughter's hypothesised function as a social lubricant (Dunbar, 2012; Dunbar et al. 2012), as disclosures are often traded as a means of developing and deepening relationships (Collins \& Miller, 1994; Derlega \& Berg, 1993). Indeed, people tend to like those to whom they disclose, as well as those who disclose to them (Collins \& Miller, 1994).

Just as primates are at risk of providing support to those who later fail to reciprocate (i.e. freeriders: Dunbar, 1999), self-disclosure is risky in that too much disclosure may lead to social exploitation (Omarzu, 2000). As a result, there is always a tension between disclosing to potential partners and avoiding manipulation (Maestripieri et al., 1992; Mehu, 2011). However, just as grooming may alleviate the tension posed by the problem of freeriders in primates (relaxing the groomed via endorphin release and hence encouraging the exchange of support: Dunbar, 2010; Goosen, 1981; Schino et al, 1988), the endorphin activation triggered 


\section{LAUGHTER'S INFLUENCE ON THE INTIMACY OF SELF-DISCLOSURE}

by laughter (Dunbar et al, 2011) may serve to assuage the tension caused by the possibility of social exploitation. After all, endogenous opioid activation triggered by laughter has been shown to have a role in regulating fear (Chaijale et al, 2013, Ipser et al, 2012; Resendez \& Aragona, 2012), and laughing has profound effects on stress reduction (Keltner \& Bonanno, 1997; Mehu, 2011). Thus, laughter, like social grooming, may serve to reduce one's sensitivity to social threat by virtue of augmenting endorphin release (Ipser et al, 2012), and this in turn, may facilitate the development of social bonds by inducing a psychological state in which the laugher is willing to begin exchanging disclosures.

Although there are currently no studies that directly address the relationship between laughter and disclosure, a recent study provides evidence that is consistent with such a link. Forgas (2011) presented participants with a video clip either from Fawlty Towers (a British comedy programme), or from a film that discussed death from cancer (My Life) in order to induce happiness or sadness (respectively). Following this, he instructed participants to imagine that they had met someone whom they found attractive and to write down five pieces of information they would like to tell this 'other' about themselves. After having rated the disclosures for intimacy, abstractness (disclosing about concrete behaviours vs. abstract traits), and valence (positivity versus negativity of content disclosed), Forgas found that those who watched the comedy clip disclosed more intimate, more positive, and more abstract information about themselves (Forgas, 2011). Forgas interpreted the difference in disclosure between the two conditions as a result of a change in mood. However, it is possible, given the choice of a comedy video to induce positive affect, that the change in disclosure was not a product of mood per se, but a result of different laughter rates in response to the two clips. Accordingly, the present study directly compares positive affect inductions involving and not involving laughter in order to determine whether laughter mediates any effects on disclosure. 


\section{LAUGHTER'S INFLUENCE ON THE INTIMACY OF SELF-DISCLOSURE}

Even if positive affect, independent of laughter, increases self-disclosure (just as it increases willingness to take risks: Trope, Ferguson, \& Raghunathan, 2001), there is reason to believe that laughter will have a greater effect on willingness to disclose than positive mood alone. Dunbar et al. (2012) found that positive affect inductions involving laughter elevated endorphin release (as measured via pain thresholds) significantly more than equivalent positive affect inductions not involving laughter. To the extent that endorphins rather than positive affect per se underpin primate social relationships (Curly \& Keverne, 2005; Dunbar, 2010; Keverne et al., 1989; cf. Spoor \& Kelly, 2004), these results imply that positive affect with laughter will be more successful than positive affect without laughter at increasing disclosure.

Another potential consideration with Forgas's procedure is that it focused entirely on how much participants were observed to disclose and so failed to consider the extent to which the participants personally felt that they disclosed. This is important, as there may be a ‘disconnect between disclosing behaviours and perceptions' (Nguyen, Bin, \& Campbell, 2012, p. 109). In other words, people in a positive mood may, in reality, reveal more than those in a sad mood, but they may feel like they disclose more when sad than when happy.

\section{The current study}

To address whether laughter has an effect on self-disclosure, the current study sought to compare the characteristics (intimacy, abstractness, and valence) of self-disclosing statements produced by those who had previously watched one of three video clips that differed in the extent to which they elicited laughter and positive affect (neutral, positive affect, and comedy). As in Forgas's (2011) study, participants in this study were asked to write down five pieces of information they would like to reveal about themselves and their statements were subsequently rated by two independent observers for intimacy, abstractness, and valence. However, unlike in Forgas's procedure, in the present study the disclosing 


\section{LAUGHTER'S INFLUENCE ON THE INTIMACY OF SELF-DISCLOSURE}

statements were also rated on the three facets of disclosure by the participants themselves (in order to address both 'actual' and perceived disclosure).

On the basis of the social lubricant theory of laughter, we predicted that people in the comedy condition would disclose more intimate information about themselves than those in either the neutral or the positive affect conditions. While we expected people in the positive affect condition to disclose more intimately than those in the neutral condition, given the influence of positive affect on willingness to take risks (Trope, Ferguson, \& Raghunathan, 2001), we also predicted that those in the comedy condition would reveal more intimate information about themselves than people in either the neutral or the positive affect conditions, since laughter, rather than positive affect per se, is hypothesised to have evolved specifically to facilitate the development of intense social bonds.

\section{Method}

\section{Design}

The experiment used a 3 x 2 between-subjects factorial design, with clip (comedy, positive affect, and neutral) and group sex (male vs. female) as the independent variables and self-reported and observer-rated disclosure (intimacy, abstractness, and valence) as the dependent variables.

\section{Participants}

Participants were 112 students (72 women, 40 men; age range 18-31; $M=22.43$ years, $S D=3.34$ years) who were compensated for their participation with $£ 10$ cash. As laughter in mixed gender groups may serve a sexual rather than a cooperative function (Mehu \& Dunbar, 2008), each group was either all male or all female in composition. Each group of four participants were randomly assigned to one of three conditions. Of the males, 12 


\section{LAUGHTER'S INFLUENCE ON THE INTIMACY OF SELF-DISCLOSURE}

individuals were assigned to the comedy condition, 16 to the neutral condition and 12 to the positive affect condition; of the females, 24 individuals were assigned to each of the three conditions. Participants were recruited via mass email to the faculties and departments of the two universities in Oxford. Participants signed up individually for the experiment.

\section{Procedure}

Four individuals arrived simultaneously at the meeting room of the social psychology laboratory, where they were welcomed by an experimenter and completed an informed consent form. None of the participants in any of these groups had met before, and all were requested not to speak to each other until asked to do so by the experimenter. Through the advertising of the study and through the participant information form, participants were led to believe that they would be given the opportunity to interact with the other participants at some later point during the study.

\section{Manipulation}

Each group of 4 participants first watched one of the10-minute mood-induction videos. The comedy film was taken from a performance by stand-up comedian Michael McIntyre (from his BBC television series Michael McIntyre's Comedy Road Show). The neutral film was taken from a golf instruction video (Paul Wilson's Golf Instruction Tips and Schools). The positive affect video was an excerpt showing lush, pleasant scenes and animals from the Jungles episode of the BBC TV series Planet Earth. These films were previously used for similar purposes by Dunbar et al (2012). The choice of a comedy clip to stimulate laughter was driven by the need to monitor Duchenne (relaxed, unforced laughter that is stimulus driven and emotionally valent) as opposed to non-Duchenne laughter (which is context-driven and emotionless). Importantly, only Duchenne laughter has been shown to increase positive affect and alleviate stress (Keltner \& Bonanno, 1997), and as a consequence only Duchenne laughter is believed to trigger endorphin release and serve the function of 


\section{LAUGHTER'S INFLUENCE ON THE INTIMACY OF SELF-DISCLOSURE}

facilitating the development of social bonds. The neutral film had previously been shown to increase positive affect, but not induce laughter (Dunbar et al. 2012).

All videos were played using Windows media player and were projected onto a SMART Board SB680 (screen size: $188 \mathrm{~cm} \times 117.2 \mathrm{~cm}$ ). Because humans do not laugh readily when watching even the funniest performances alone (Freedman \& Perlick, 1979; Provine, 1996), and because laughter is 30 times more likely to occur in social contexts than when alone (Provine, \& Fischer, 1989), all subjects were tested in groups of four.

\section{Manipulation checks}

Laughter rates and funniness. Since it was important that the experimenter left the room during the manipulation (Chapman, 1976) and since people are poor judges of how much they laugh (Vettin \& Todt, 2003; 2004), laughter to the video clips was recorded by a small audio recorder, discretely placed behind the participants. At the end of the study, two raters independently counted the number of laughs recorded for the group for each 10 minute session. These ratings were highly consistent (Cohen's kappa $=.82$ ). For analysis, we averaged the raters' scores. Participants also rated how funny they found the clip on a scale of 1 (not at all) to 5 (a great deal).

PANAS. Immediately after the clip, the participants' emotional state was assessed using the positive and negative affect schedule (PANAS). The PANAS is a 20 item selfreport measure of positive and negative affect developed by Watson, Clark and Tellegen (1988). The PANAS requests that participants indicate the extent to which they are currently experiencing a range of positive and negative emotions on a scale of 1 (very slightly or not at all) to 5 (extremely). There are 10 items that relate to positive affect (e.g. enthusiastic, interested, and excited) and 10 items that relate to negative affect (e.g. upset, ashamed, and irritable). 


\section{LAUGHTER'S INFLUENCE ON THE INTIMACY OF SELF-DISCLOSURE}

\section{Self-disclosure task}

After the manipulation checks, in an ostensibly unrelated experiment on "social communication", participants were instructed to sit in separate corners of the room and pick up their (randomly assigned) piece of differently coloured card (red, blue, green, or yellow) and show it to the other participants. Participants were instructed to remember which individuals held which coloured cards, before facing away from each other to individually complete a questionnaire. As part of the questionnaire, participants were instructed to compose a message for one of the members of the group as follows:

Of the other people present whilst you watched the clip, please compose a message for the person holding the [ ] card so that they can get to know you better. Write down on the sheet provided the five pieces of information you would like to say about yourself to this person.

Please use the same words you would use in the actual conversation. You will subsequently have the opportunity to interact with this person.

This was adapted from Forgas's (2011) self-disclosure task. However, while Forgas requested that participants targeted their message to a hypothetical other, with whom they were instructed to imagine having a conversation, the present study asked participants to compose a message for someone they have just met and believe that they are about to interact with. This was intended to increase the ecological validity of the task.

All participants composed a message for someone who did not compose a message for them. Coupled with the fact that participants individually completed the questionnaire, this all but eliminated the possibility of the participants attempting to signal to their partners that they were writing a message for each other and hence manipulate their partner's desire to disclose (e.g. catch their partner's eye and smile).

\section{Dependent measures}




\section{LAUGHTER'S INFLUENCE ON THE INTIMACY OF SELF-DISCLOSURE}

Following the disclosure task, participants then rated their five self-disclosing statements on 10-point scales for intimacy, abstractness, and valence. These constructs were defined (as in Forgas, 2011) to the participants as follows: (a) intimacy: revealing more or less personal details; (b) abstractness: information about specific versus general, abstract characteristics (e.g., disclosing about concrete behaviours vs. abstract traits); and (c) valence: positivity versus negativity of content disclosed (e.g., revealing successes vs. failures).

The five disclosing statements were also rated on the same dimensions (i.e. intimacy, abstractness and valence) on 10-point scales by two independent raters blind to the experimental condition. The constructs were defined to the raters in the same way that they were defined to the participants. As the two raters showed acceptable levels of agreement across each dimension of disclosure (Cohen's kappa $=.76$ ) their ratings were averaged for further analysis.

\section{Control measures}

Participants were also requested to provide information about their own traits, which have previously been shown to modify self-disclosure in interactions (Greene, Derlega, \& Mathews, 2006). To this end, participants were asked to complete the 10 item personality inventory (TIPI); a psychometrically reliable and valid instrument for testing openness, conscientiousness, agreeableness, extraversion, and neuroticism (Gosling, Rentfrow, \& Swann, 2003).

\section{Debriefing}

Debriefing featured a series of open-ended questions about what participants thought about the study. Responses suggested that participants had not been aware of the manipulations. 


\section{Results}

\section{Manipulation checks}

Laughter rates and funniness. To establish that laughter rates differ across the conditions in the way predicted, we tested for an effect of video type on laughter whilst watching the video. There was a main effect of condition on the extent to which participants laughed during the $\operatorname{clip}\left(F(2,109)=28.45, p<0.001, \eta^{2}=.34\right)$ and on the extent to which they found the clip funny $\left(F(2,109)=17.72, p<0.001, \eta^{2}=.25\right)$. Scheffé post hoc tests confirmed that both laughter and rated funniness were (i) significantly higher in the comedy condition than in all other conditions $(p<0.001)$ and (ii) that there were no significant differences in laughter and rated funniness between the neutral and the positive affect conditions $(p>0.05)$. (Since participants completed the study in groups, the analyses were repeated at group level using aggregated data; the pattern results were identical in all cases, n=28.) For the means and standard deviations see table 1.

Mood and positive affect. To check whether the comedy and positive affect conditions increased positive affect more than the neutral condition, and to confirm that the comedy and the positive affect video were matched in their effect on mood, we examined the effect of video type on positive and negative affect. Contrary to expectation, there was no main effect of video type on overall positive affect $\left(F(2,108)=1.21, p>.05, \eta^{2}=.02\right)$ or overall negative affect $\left(F(2,109)=.87, p>0.05, \eta^{2}=.02\right)$, although the results are in the predicted direction. However, there was a main effect of video clip on how interested participants reported to be feeling (condition: $\left.F(2,109)=6.04, p<.05, \eta^{2}=.10\right)$. Scheffé post hoc tests revealed that interest was (i) significantly higher in the comedy and positive affect condition than in the neutral condition $(p<0.05)$ and (ii) that there was no significant difference in reported interest between the comedy and the positive affect conditions ( $p>$ 


\section{LAUGHTER'S INFLUENCE ON THE INTIMACY OF SELF-DISCLOSURE}

0.05). Thus, at least one aspect of positive affect was influenced as intended by the manipulation. For the means and standard deviations see Table 1.

Table 1

Mean and Standard Deviation for Laughter, funniness and affect as a function of condition

\begin{tabular}{|c|c|c|c|c|c|c|}
\hline \multirow[b]{2}{*}{ Measure } & \multicolumn{2}{|c|}{ Comedy $(n=36)$} & \multicolumn{2}{|c|}{ Positive affect $(n=36)$} & \multicolumn{2}{|c|}{ Neutral $(n=40)$} \\
\hline & $M$ & $S D$ & $M$ & $S D$ & $M$ & $S D$ \\
\hline Laughter & 17.22 & 17.43 & .44 & .84 & 2.80 & 4.66 \\
\hline Funniness & 3.03 & 1.11 & 1.75 & .81 & 1.95 & 1.01 \\
\hline $\begin{array}{l}\text { Overall } \\
\text { Positive } \\
\text { Affect }\end{array}$ & 2.47 & .60 & 2.63 & .61 & 2.41 & .70 \\
\hline $\begin{array}{c}\text { Overall } \\
\text { Negative } \\
\text { Affect }\end{array}$ & 1.18 & .25 & 1.19 & .22 & 1.25 & .30 \\
\hline Interest & 3.42 & .77 & 3.50 & .94 & 2.89 & .85 \\
\hline
\end{tabular}

\section{The effect of laughter on disclosure}

The relationship between self-report and observer-rated disclosure. To test

whether self-reported disclosure and observer-rated disclosure are independent measures of disclosure, the three disclosure dimensions were correlated across these two forms of measurement. Self-reported valence and observer-rated valence correlated significantly $(r$ $(112)=.27, p<.05)$ as did self-reported intimacy and observer rated intimacy $(r(112)=.20$, $p<.05$ ). However the modest size of these correlations suggests that raters and disclosers had partly discrepant perceptions of these dimensions. Further, the correlation between self- 


\section{LAUGHTER'S INFLUENCE ON THE INTIMACY OF SELF-DISCLOSURE}

reported abstractness and observer-rated abstractness was non-significant and close to zero $(r$ $(112)=.06, p>.05)$.

Laughter and self-reported disclosure. Considering self-reported disclosure only, the three facets of disclosure (intimacy, abstractness and valence) were correlated with one another to test their independence. There were no significant relationships between any of the three facets of self-reported disclosure $(p>0.05)$.

We ran a multivariate analysis of variance (MANOVA) with the three self-rated facets of disclosure (i.e. intimacy, abstractness and valence) as the dependent variables, with gender (male/ female) and condition (comedy, positive affect and neutral) as the two independent variables. There was no main effect of gender (Pillai's trace $V=.059, F(3,104)$ $\left.=2.190, p>.05, \eta^{2}=.06\right)$ or of condition $\left(V=.051, F(6,210)=.924, p>.05, \eta^{2}=.03\right)$, nor was there an interaction effect between the two $\left(V=.073, F(6,210)=1.32, p>.05, \eta^{2}=\right.$ .04). These results held, even when separately and concurrently controlling for the effects of personality traits that have previously been shown to influence disclosure rates (i.e. extraversion, neuroticism and openness).

Laughter and observer-rated disclosure. As with self-rated disclosure, we first tested whether the different facets of observer-rated disclosure (intimacy, abstractness, and valence) were indeed independent of one another. Only observer-rated intimacy and abstractness were significantly related $(r(112)=.47, p<0.01)$.

We then ran a second MANOVA with each of the three observer-rated intimacy, abstractness and valence facets of disclosure as the dependent variables and gender (male/ female) and condition (comedy, positive affect and neutral) as the two independent variables. The multivariate main effect of condition was significant $(V=.240, F(6,210)=4.64, p<$ $\left..05, \eta^{2}=.12\right)$ but the main effect of gender $\left(V=.037, F(3,104)=1.33, p>.05, \eta^{2}=.04\right)$ and 


\section{LAUGHTER'S INFLUENCE ON THE INTIMACY OF SELF-DISCLOSURE}

the interaction effect $\left(V=.047, F(6,210)=, p>.05, \eta^{2}=.02\right)$ were non-significant. There were also significant univariate main effects of condition for observer-rated intimacy $(F$ $\left.(2,106)=5.81, p<.05, \eta^{2}=.10\right)$ and abstractness $\left(F(2,106)=6.187, p<.05, \eta^{2}=.11\right.$, see

Figure 1), but not on observer-rated valence $\left(F(2,106)=1.17, p>.05, \eta^{2}=.02\right)$.

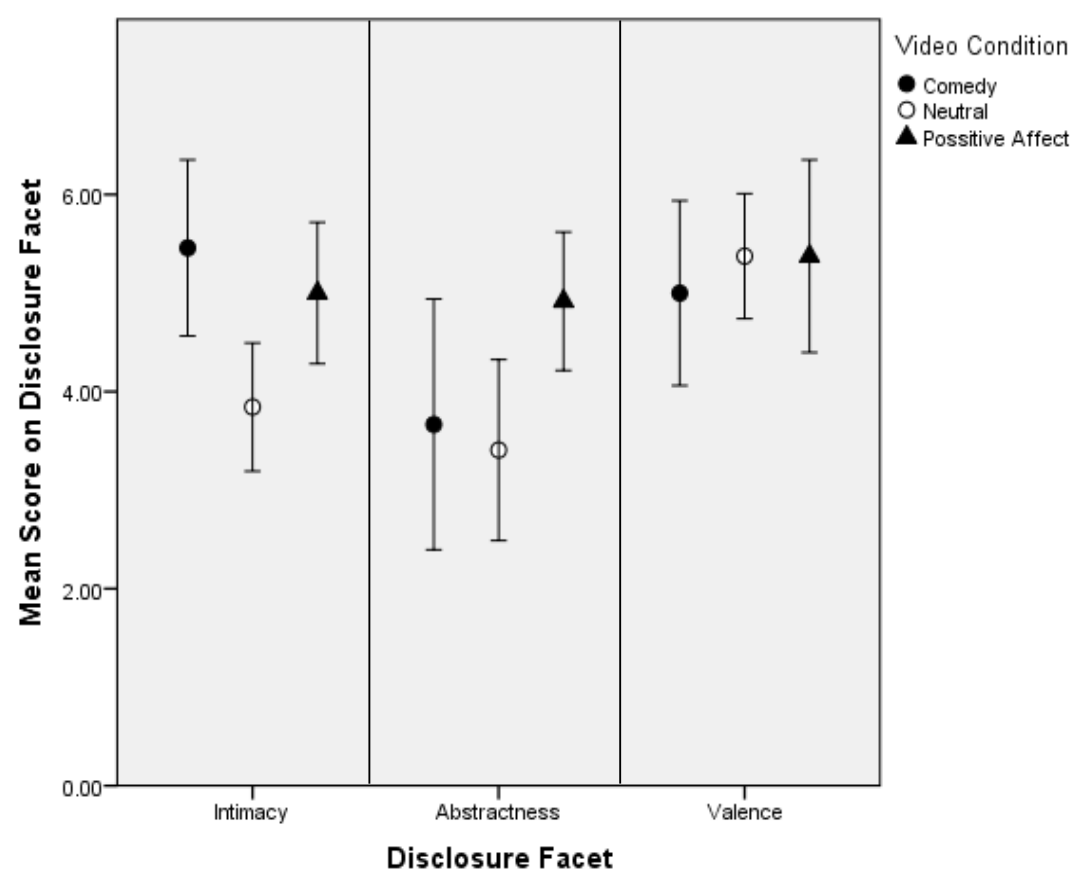

Figure 1: Mean score (+s.e.) for each observer-rated disclosure facet (intimacy, abstractness and valence) as a function of video condition (comedy, neutral and positive affect).

Scheffé post hoc tests for observer-rated intimacy revealed that (i) intimacy was significantly higher in the comedy $(M=5.24, S D=1.72)$ than in the neutral $(M=4.04, S D=$ 1.47) condition $(p<0.05)$, (ii) that the difference in observer-rated intimacy between the comedy and the positive affect $(M=4.54, S D=1.54)$ condition was non-significant $(p>$ 


\section{LAUGHTER'S INFLUENCE ON THE INTIMACY OF SELF-DISCLOSURE}

0.05 ) and (iii) that the difference in observer-rated intimacy between the neutral and the positive affect condition was non-significant $(p>.05)$. Scheffé post hoc tests for observerrated abstractness revealed that (i) the difference in observer-rated abstractness between the comedy condition $(M=4.07, S D=1.24)$ and the neutral condition $(M=3.64, S D=1.63)$ was non-significant $(p>.05)$ and (ii) that abstractness was significantly higher in the positive affect condition $(M=5.06, S D=1.24)$ than in the comedy or the neutral conditions $(p<$ 0.05). Separately and concurrently controlling for the effects of personality traits that have previously been shown to influence disclosure rates (i.e. extraversion, neuroticism and openness) did not affect the pattern of significant results.

\section{Discussion}

The present finding that observer-rated intimacy increased in the comedy condition relative to the neutral condition supports Forgas's (2011) result that people who watched a comedy clip were more likely to disclose more intimate information than those who watched a sad clip. However, while Forgas attributed the difference in disclosure to a change in affect, the present study found that the induction of positive affect alone (at least in terms of manipulating interest) was insufficient to produce significant changes in the intimacy of disclosure. Although merely inducing positive affect (or at least one aspect of it) did indeed increase the personal intimacy of disclosure relative to the neutral condition, only the comedy condition did so significantly. This suggests that Forgas's results may have been a consequence of disparate laughter rates, rather than changes in mood per se. Indeed, the fact that the present study failed to induce significant changes in overall positive affect (beyond interest) in the comedy, neutral, and positive affect conditions, argues against the interpretation that a change in mood is responsible for the differences in disclosure intimacy. After all, there was no significant difference in overall positive or negative affect between the comedy, neutral, and positive affect conditions, but the three conditions did differ 


\section{LAUGHTER'S INFLUENCE ON THE INTIMACY OF SELF-DISCLOSURE}

significantly on the amount of laughter they elicited and the intimacy of the disclosures they provoked (with comedy generating significantly more laughs than the other two conditions, as well as more intimate disclosures).

One likely reason why the comedy condition elicited significantly more intimate disclosures than the neutral condition is that the higher laughing rate led to an increase in endorphin activation. Given laughter's effect on endorphin release (Dunbar et al, 2012) and the role of endorphins in the formation of social bonds (Machin \& Dunbar, 2011), laughter, through the psychopharmacological effects of endorphins, may have increased willingness to disclose intimate information. As the effect of endorphins may be limited to behaviours associated with social bonding (Machin \& Dunbar, 2011), the present finding that the abstractness and valence of disclosures were not significantly increased in the comedy condition relative to the neutral or the positive affect conditions is particularly interesting and may reflect their lack of importance in relationship development. Certainly, when we think about disclosure as a tool for building relationships, how abstract or positive disclosures are seems far less important than their personal intimacy (Collins \& Miller, 1994; Derlega \& Berg, 1993). Similarly, laughter may have influenced observer-rated but not self-rated disclosure intimacy, as the former may be more critical for relationship development. In other words, the extent to which one feels one has disclosed may not be as important for relationship development as the extent to which others judge one to have disclosed (Nguyen, Bin \& Campbell, 2012; Schiffrin et al, 2010).

Precisely how endorphins work to increase disclosure intimacy is a question for future research. Although it has been suggested that endorphins influence social bonding via their effect on mood (Dunbar et al, 2012), the fact that the present study found no effect of increasing positive mood on disclosure intimacy suggests that this may be incorrect. Indeed, the comedy and the positive affect conditions were matched on feelings of interest and 


\section{LAUGHTER'S INFLUENCE ON THE INTIMACY OF SELF-DISCLOSURE}

overall positive and negative affect, so any difference between the two conditions cannot be attributed to discrepancies in emotional state - at least not as measured by PANAS.

One possibility is that endorphins influence disclosure intimacy (and hence facilitate the development of social bonds) by allowing one to shift attention away from oneself. Since excessive self-focus (i.e. a preoccupation with how the self appears to others and a heightened attention to internally generated information) increases access to negative attitudes and interferes with performance in social situations (Ickes, Wicklund, \& Ferris, 1973; Mor \& Winquist, 2002), endorphins may serve to facilitate interaction through reducing self-directed attention. This makes sense, given opiates' main effect of inducing relaxation. Without laughter, people in the neutral condition may have been more attuned to their own concerns about disclosing too much information to others and coming across as 'weird' or unlikable, while, conversely, those in the comedy condition, by virtue of their laughter, may have been more attentive to the demands of the situation and thus less likely to pay attention to such thoughts.

That endorphins may increase disclosure intimacy by shifting attention away from the self, suggests an alternative though not necessarily mutually exclusive explanation for why laughter influenced observer-rated but not self-rated disclosure intimacy; namely, that laughter reduced self-focus, and that this in turn reduced people's awareness of how intimately they were disclosing. This raises a series of intriguing questions for further study. First, it predicts that manipulating self-focus will affect the extent to which there is a discrepancy between self-rated and observer-rated disclosure. Second, it suggests the possibility that laughter will lead people to be inaccurate in their judgments of their own disclosure, yet accurate in the extent to which they judge people to have disclosed to them. And finally, it suggests that people with psychiatric disorders characterised by excessive self- 


\section{LAUGHTER'S INFLUENCE ON THE INTIMACY OF SELF-DISCLOSURE}

focus, such as social anxiety disorder (Clark \& Wells, 1995; Ingram 1990), might benefit from practicing laughter when in the company of others.

Another result of the present study is that people in the positive affect condition disclosed significantly more abstractly than those in either the neutral or comedy conditions. As participants in the positive affect manipulation were no higher on positive affect scores than those in other conditions, we may infer that something about the positive affect condition, besides its effect on mood, caused people to disclose more abstractly. When thinking about the differences between the videos used in the positive affect condition and the videos used in the neutral and comedy conditions, the possibility arises that the content of the positive affect video raised more abstract themes than the more specific observations of Michael McIntyre (comedy video) or the more specific advice about golf provided by Paul Wilson (neutral video). It is therefore possible that participants may therefore have been primed in the positive affect condition, via exposure to abstract content, to subsequently reveal more abstract disclosures (Gilead, Liberman, \& Maril, 2013).

The present study also found no difference between the neutral condition and the positive affect and comedy conditions on self-rated or observer-rated disclosure positivity. While it is possible that this result reflects a true lack of an effect of comedy and positive affect on disclosure positivity, it is also conceivable that this result is a product of the strength of the present manipulation of mood. Notably, both the positive affect and comedy conditions did not differ significantly from the neutral condition on overall positive or negative affect. This suggests that both these conditions were not very successful in influencing the participant's emotional state, and consequently, that the change in mood in the comedy and positive affect conditions was insufficient to cause any differences in disclosure positivity between people in these conditions and people in the neutral condition. 


\section{LAUGHTER'S INFLUENCE ON THE INTIMACY OF SELF-DISCLOSURE}

While the present study improved upon the ecological validity of Forgas's (2011) self-disclosure task by having participants compose a message for another participant as opposed to an imaginary other, it is possible that the act of disclosing via a written message does not reflect disclosure in a conversational context. One reason may relate to communication mode richness (Daft \& Lengel, 1986). In comparison to communicating face to face (FTF), sending a message to another via written text provides a substantial reduction in communication channels (notably facial expressions and voice intonations). If people prefer to communicate difficult concepts and emotionally sensitive information via 'rich' media (Daft, Lengel, \& Trevino, 1987), it is possible that the current lack of an effect of laughter on the abstractness and the valence of disclosures is a consequence of the communication medium, rather than a lack of an effect of laughter per se. Future research should therefore address whether similar results are obtained when participants interact with one another FTF as opposed to via written text.

Regardless of whether disclosure via the written word corresponds to disclosure in a conversational context, the present study's use of text as a medium for participants' disclosures has value in itself. With the rise of social networking sites (SNS) such as Facebook and Twitter, many people now frequently reveal a significant amount of personal information via text (Coleman, Paternite \& Sherman, 1999; Naaman, Boase \& Lai, 2010). While it may not be the 'richest' medium for communicating personal information (Daft \& Lengel, 1986), a growing number of people use it to do so every day (Kock, 2005). To the extent that the present study's disclosure task mimics communication via SNS, one may wonder how applicable the current result of a 'disconnect' between actual and perceived disclosure is to interaction via SNS.

One reason why self-reported and observer-rated disclosure differed in the present task is that the disclosures did not involve immediate and continuous feedback such as that 


\section{LAUGHTER'S INFLUENCE ON THE INTIMACY OF SELF-DISCLOSURE}

provided by facial expressions (Parkinson, 2008). Like people disclosing on SNS, people in the present study were unable to observe another's responses to their disclosures, both as they were producing them and after they had been produced. This may have distorted selfjudgments about how much one had disclosed. This possibility awaits testing.

Another limitation of the present design is that we only recorded overall laughter for the group and did not record how much each individual within a group laughed. This is important, as people in the present study may have disclosed more intimately in the comedy condition, not because they laughed more per se, but because they were asked to compose a message to someone they had previously seen laughing. In other words, it may not be the act of laughing which facilitates disclosure, but the perception of another laughing which makes people more willing to reveal personal information when subsequently asked to communicate with the laugher. This might reflect the fact that a shared sense of humour seems to be a powerful cue of shared interests and potential friendship (Curry \& Dunbar 2012), and may thus signal the potential for greater intimacy.

In all likelihood, however, the relationship between laughter and disclosure intimacy in the present study is a result of a combination of both laughing oneself and perceiving others laughing. Indeed, the well-known contagion effect of laughter (Provine, 2000; Dunbar et al. 2012) suggests that the two processes work in tandem, with others' laughter generally leading to one's own laughter and own laughter typically inducing more laughter in others. Thus, own laughter and others' laughter likely merge to augment disclosure overall. Nevertheless, future research measuring laughter rates on an individual basis is needed to establish more precisely the way laughter facilitates self-disclosure.

The principal goal of the present research was to test the social lubricant theory of laughter by asking whether laughter increases the probability that people will disclose more intimate information about themselves, and hence facilitate the formation of social bonds 


\section{LAUGHTER'S INFLUENCE ON THE INTIMACY OF SELF-DISCLOSURE}

between people. Through controlled experimental conditions, we found support for the role of laughter in encouraging more intimate disclosures to strangers but not more abstract or positive disclosures. Of particular interest was the fact that this effect was limited to observer-ratings of disclosure and not self-ratings of disclosure. This was suggested to be in line with the notion that laughter is linked specifically to fostering behaviours that encourage relationship development, since observer-ratings of disclosure may be more important for relationship development than how much one feels like one is disclosing. These results suggest that laughter should be a serious topic for those interested in the development of social relationships.

Acknowledgements: AG was supported by the Crewe Graduate Scholarship, Lincoln College Oxford. RD's research is supported by a European Research Council Advanced grant.

\section{References.}

Bachorowski, J.-A. \& Owren, M. J. (2001). Not all laughs are alike: voiced but not unvoiced laughter readily elicits positive affect. Psychological Science, 12, 252-257.

Berman, M.G., Jonides, J., \& Kaplan, S. (2008). The cognitive benefits of interacting with nature. Psychological Science, 19, 1207-1212.

Berto, R. (2005). Exposure to restorative environments helps restore attentional capacity. Journal of Environmental Psychology, 25, 249-259.

Chaijale, N. N., Curtis, A. L.,, Wood, S. K.,, Zhang, X.,, Bhatnagar, S.,, Reyes, B. A. S., Bockstaele, E.V., \& Valentino, R. J. (2013). Social stress engages opioid regulation of locus coeruleus norepinephrine neurons and induces a state of cellular and physical opiate dependence. Neuropsychopharmacology, 38, 1-11. 


\section{LAUGHTER'S INFLUENCE ON THE INTIMACY OF SELF-DISCLOSURE}

Chapman, A. J. (1976). Social aspects of humorous laughter. In A. J. Chapman \& H. C. Foot

(Eds.), Humour and laughter: theory, research and applications (pp. 155-185).

London: Wiley.

Clark, D. M., \& Wells, A. (1995). A cognitive model of social phobia. In R. G. Heimberg, M.

Liebowitz, D. A. Hope, \& F. Schneier (Eds.), Social phobia: diagnosis, assessment and treatment. New York: Guilford.

Coleman, L. H., Paternite, C. E., \& Sherman, R. C. (1999) A re-examination of deindividuation in synchronous computer-mediated communication. Computers in Human Behaviour, 15, 51-65.

Collins, N. L., \& Miller, L. C. (1994). Self-disclosure and liking: a meta-analytic review. Psychological Bulletin, 116, 457-475.

Curley, J. P., \& Keverne, E. B. (2005). Genes, brains and mammalian social bonds. Trends in Ecology \& Evolution, 20, 561-567.

Curry, O. \& Dunbar, R.I.M. (2013). Sharing a joke: the effects of a similar sense of humor on affiliation and altruism. Evolution and Human Behavior 34: 125-129.

Daft, R.L., \& Lengel, R. H. (1986). Organizational information requirements, media richness, and structural design. Management Science, 32, 554-571.

Daft, R. L., Lengel, R. H., \& Trevino, L. K. (1987). Message equivocality, media selection and manager performance: Implications for information systems. MIS Quarterly, 11, $355-366$.

Derlega, V., \& Berg, J. H. (1993). Self-disclosure. Newbury Park, CA: Sage.

Dunbar, R.I.M. (1999). Culture, honesty and the freerider problem. In: R.I.M. Dunbar, C. Knight \& C. Power (eds) The evolution of culture (pp. 194-213). Edinburgh University Press: Edinburgh. 


\section{LAUGHTER'S INFLUENCE ON THE INTIMACY OF SELF-DISCLOSURE}

Dunbar, R. I. M. (2010). The social role of touch in humans and primates: Behavioural function and neurobiological mechanisms. Neuroscience and Biobehavioral Reviews, 34, 260-268.

Dunbar, R. I. M. (2012). Bridging the bonding gap: the transition from primates to humans. Philosophical Transactions of the Royal Society B: Biological Sciences, 367, 18371846.

Dunbar, R. I. M., Baron, R., Frangou, A., Pearce, E., van Leeuwen, E. J. C., \& Stow, J., et al (2012). Social laughter is correlated with an elevated pain threshold. Proceedings of the Royal Society B: Biological Sciences, 279, 1161-1167.

Forgas, J. P. (2011). Affective influences on self-disclosure: mood effects on the intimacy and reciprocity of disclosing personal information. Journal of Personality and Social Psychology, 100, 449-461.

Freedman, J. L. \& Perlick, D. (1979). Rowing, contagion and laughter. Journal of Experimental Social Psychology, 15, 295-303.

Gilead, M., Liberman, N., \& Maril, A. (2013). From mind to matter: neural correlates of abstract and concrete mindsets. Social Cognitive and Affective Neuroscience, 8, 341350.

Goosen, C. (1981). On the function of allogrooming in Old-World monkeys. In A. B.

Chiarelli \& R. S. Corruccini (Eds.), Primate behaviour and sociobiology (pp. 110120). Springer, Berlin.

Gosling, S. D., Rentfrow, P. J., \& Swann, W. B. (2003). A very brief measure of the Big-Five personality domains. Journal of Research in Personality, 37, 504-528.

Greene, K., Derlega, V. J., \& Mathews, A. (2006). Self-disclosure in personal relationships. In A. Vangelisti \& D. Perlman (Eds.), Cambridge handbook of personal relationships (pp. 409-427). Cambridge, England: Cambridge University Press. 


\section{LAUGHTER'S INFLUENCE ON THE INTIMACY OF SELF-DISCLOSURE}

Ickes, W. J., Wicklund, R. A., \& Ferris, C. B. (1973). Objective self-awareness and selfesteem. Journal of Experimental Social Psychology, 9, 202-219.

Ingram, R. E. (1990). Self-focused attention in clinical disorders: review and a conceptual model. Psychological Bulletin, 107, 156-176.

Ipser, J.C., Terburg., Syal, S., Phillips, N., Solms, M., Panksepp, J., Malcolm-Smith, S., Thomas, K., Stein, D. J., \& Honk, J. V. (2013). Reduced fear-recognition sensitivity following acute buprenorphine administration in healthy volunteers, Psychoneuroendocrinology, 38, 166-170.

Keltner, D., \& Bonanno, G. A. (1997). A study of laughter and dissociation: Distinct correlates of laughter and smiling during bereavement. Journal of Personality and Social Psychology, 73, 687-702.

Keverne, E. B., Martensz, N., Tuite, B. (1989). Beta-endorphin concentrations in cerebrospinal fluid of monkeys are influenced by grooming relationships. Psychoneuroendocrinology, 14, 155-161.

Kock, N. (2005). Media richness or media naturalness? The evolution of our biological communication apparatus and its influence on our behaviour toward communication tools. IEEE Transactions on Professional Communication, 48, 117-130.

Li, N. P., Griskevicius, V., Durante, K. M., Jonason, P. K., Pasisz, D. J., \& Aumer, K. (2009). An evolutionary perspective on humor: sexual selection or interest indication? Social Psychology Bulletin, 35, 923.

Machin, A. J., \& Dunbar, R. I. M. (2011). The brain opioid theory of social attachment: a review of the evidence. Behaviour, 148, 985-1025.

Maestripieri, D., Schino, G., Aureli, F., \& Troisi, A. (1992). A modest proposal: displacement activities as an indicator of emotions in primates. Animal Behaviour, 44, 967-979. 


\section{LAUGHTER'S INFLUENCE ON THE INTIMACY OF SELF-DISCLOSURE}

Mehu, M. (2011). Smiling and Laughter in Naturally Occurring Dyadic Interactions:

Relationship to Conversation, Body Contacts, and Displacement Activities. Human Ethology Bulletin, 26, 10-28.

Mehu, M., \& Dunbar, R.I.M. (2008). Naturalistic observations of smiling and laughter in human group interactions. Behaviour, 145, 1747-1780.

Mor, N., \& Winquist, J. (2002). Self-Focused Attention and Negative Affect: A MetaAnalysis Psychological Bulletin, 128, 638-662.

Naaman, M., Boase, J., \& Lai, C. H. (2010). Is it really about me? Message content in social awareness streams. Proceedings of the 2010 ACM conference on computer supported cooperative work (association for computing machinery) (pp. 189-192) Savannah, GA.

Nguyen, M., Bin, Y. S., \& Campbell, A. (2012). Comparing Online and Offline SelfDisclosure: A Systematic Review. Cyberpsychology, Behaviour and Social Networking, 15, 103-111.

Omarzu, J. (2000). A disclosure decision model: determining how and when individuals will self-disclose. Personality and Social Psychology Review, 4, 174-185.

Owren, M. J. \& Bachorowski, A. (2003). Reconsidering the evolution of non-linguistic communication: the case of laughter. Journal of Nonverbal Behaviour. 27, 183-200.

Parkinson, B. (2008). Emotions in direct and remote social interaction: Getting through the spaces between us. Computers and Human Behaviour, 24, 1510-1529.

Provine, R. (1996). Laughter: a scientific investigation. London, UK: Faber \& Faber.

Provine, R. R. \& Fischer, K. R. (1989). Laughing, smiling, and talking: relation to sleeping and social context in humans. Ethology, 83, 295-305.

Resendez, S. L., \& Aragona, B. J. (2012). Aversive motivation and the maintenance of monogamous pair bonding. Nature Reviews Neuroscience, 24, 51-60. 


\section{LAUGHTER'S INFLUENCE ON THE INTIMACY OF SELF-DISCLOSURE}

Schiffrin, H., Edelman, A., Falkenstern, M., \& Stewart, C. (2010). The associations among computer-mediated communication, relationships, and well-being. Cyberpsychology, Behavior, and Social Networking, 13, 299-306.

Schino, G., Scucchi, S., Maestripieri, D., \& Turillazzi, P.G. (1988). Allogrooming as a tension-reduction mechanism: a behavioural approach. American Journal of Primatology, 16, 43-50.

Spoor, J. R. \& Kelly, J. R. (2004). The evolutionary significance of affect in groups: communication and group bonding. Group Processes and Intergroup Relations, 7 , $398-412$.

Tennessen, C. M., \& Cimprich, B. (1995). Views to nature: effects on attention. Journal of Environmental Psychology, 15, 77-85.

Trope, Y., Ferguson, M., \& Raghunathan, R. (2001). Mood as a resource in processing selfrelevant information. In J. P. Forgas (Ed.), The handbook of affect and social cognition (pp. 256-274). Mahwah, NJ: Erlbaum.

Watson, D., Clark, L. A., \& Tellegen, A. (1988). Development and validation of brief measures of positive and negative affect. Journal of Personality and Social Psychology, 54, 1063-1070.

Vettin, J. \& Todt, D. (2004). Laughter in conversation. Journal of Nonverbal Behaviour, 28, 93-115. 